\title{
Noise-Adjusted Subspace Discriminant Analysis for Hyperspectral Imagery Classification
}

\author{
Wei Li, Member, IEEE, Saurabh Prasad, Member, IEEE, and James E. Fowler, Senior Member, IEEE
}

\begin{abstract}
Linear discriminant analysis (LDA) is a popular approach for dimensionality reduction for pattern classification; however, its performance is often degraded when samples are too few, particularly when the dimensionality of the input feature space is excessively high. The classic solution to the smallsample-size problem is to implement LDA in a principal component (PC) subspace, i.e., a strategy known as subspace LDA. This latter approach is extended by coupling LDA and noise-adjusted HSI analysis in order to provide noise-robust feature extraction and classification of high-dimensional data. An extension of the proposed approach in a kernel-induced space is also studied. The resulting noise-adjusted subspace discriminant analysis is evaluated using hyperspectral imagery, with experimental results demonstrating that the proposed approach provides not only superior classification performance, as compared with traditional methods, but also effective dimensionality reduction for classification even in the presence of noise.
\end{abstract}

Index Terms-Feature extraction, hyperspectral classification, linear discriminant analysis (LDA), noise-adjusted principal component analysis (NA-PCA).

\section{INTRODUCTION}

$\mathbf{P}$ RINCIPAL component analysis (PCA) and linear discriminant analysis (LDA) are popular dimensionality reduction techniques [1] for hyperspectral imagery (HSI) classification. In general, for classification tasks, PCA-based dimensionality reduction is suboptimal compared with LDA-based methods [2]. In essence, this suboptimality is due to the fact that PCA-based methods aim to find projections with minimal reconstruction error, whereas LDA-based methods seek to find projections that preserve the discriminant information. However, under smallsample-size (SSS) conditions [3], LDA and its variants can potentially fail, owing to ill-conditioned statistical estimates, i.e., particularly when the dimensionality of the input feature space is very high, as is the case for HSI when the number of available samples is even smaller than the dimensionality.

Manuscript received February 28, 2012; revised June 29, 2012, September 2, 2012, and October 15, 2012; accepted October 16, 2012. Date of publication March 7, 2013; date of current version October 10, 2013. This work was supported in part by the University of Houston Startup Funding and in part by the National Science Foundation under Grant CCF-0915307.

W. Li is with the Center for Spatial Technologies and Remote Sensing, University of California, Davis, CA 95616 USA (e-mail: liwei089@ieee.org).

S. Prasad is with the Department of Electrical and Computer Engineering, University of Houston, Houston, TX 77204-4005 USA (e-mail: saurabh.prasad@ieee.org).

J. E. Fowler is with the Department of Electrical and Computer Engineering and the Geosystems Research Institute, Mississippi State University, Starkville, MS 39762 USA (e-mail: fowler@ece.msstate.edu).

Color versions of one or more of the figures in this paper are available online at http://ieeexplore.iee.org.

Digital Object Identifier 10.1109/LGRS.2013.2242042
A few solutions (e.g., in [1], [3], and [4]) have been proposed for the SSS problem associated with LDA. A popular approach is to use PCA-based dimensionality reduction as a preprocessing to LDA. That is, PCA is first employed to reduce the dimensionality of the input data from $d$ to a certain value $d^{\prime}$. (Yang and Yang [4] suggest that an appropriate value for $d^{\prime}$ is the rank of the total scatter matrix.) Then, LDA is performed in the low-dimensional PCA subspace. The essence of this approach, which is referred to as subspace LDA (SLDA), is that the PCA transformation discards the null space of rankdeficient scatter matrices so that the LDA formulations remain well conditioned.

In SLDA, traditional PCA is based on the maximum variance criterion, which compacts data into the first $d^{\prime}$ principal components (PCs). However, it is well known that both signal and noise can contribute to data variance. In fact, a criterion based on SNR is more appropriate for practical dimensionality reduction, As a consequence, in this letter, noise-adjusted SLDA (NA-SLDA) is considered for effective and noise-robust feature extraction of HSI. We note that noise-adjusted PCA (NA-PCA) [5], [6] has been previously developed and studied for effective dimensionality reduction of high-dimensional data. In fact, NA-PCA is identical to a maximum-noise-fraction (MNF) transformation [7], which employs several different methods to estimate the noise variance. In this letter, NA-PCA estimates the noise variance using the approach developed, particularly for HSI in [8].

For many practical applications, linear feature extraction methods have a limitation in that they fail to effectively capture the nonlinearly clustered data structure, which results from complex HSI distributions. An effective solution for this limitation is to extend the transformation in a kernel-induced space. The central idea behind kernel-based methods is to map the data from the original space into a kernel-induced feature space via a kernel function. In remote sensing settings, kernel discriminant analysis (KDA), kernel PCA (KPCA), and support vector machines (SVMs) have been studied and shown to be effective for anomaly detection and pattern classification tasks [9].

In this letter, in an effort to preserve more signal information while suppressing noise in the first $d^{\prime}$ PCs, we employ an SNR metric instead of the maximum variance criterion, as used in traditional PCA. It is hence expected that LDA in an NA-PCA feature subspace will improve classification performance. In this letter, we study the resulting NA-SLDA approach to dimensionality reduction in terms of its efficacy in retaining the most useful class-specific information under SSS conditions for HSI-classification problems. We also extend NA-PCA and the proposed NA-SLDA in a kernel-induced space. 


\section{Dimensionality Reduction}

Consider a data set with training samples $\mathbf{X}=\left\{\mathbf{x}_{i}\right\}_{i=1}^{n}$ in $\mathbb{R}^{d}$ ( $d$-dimensional feature space) and class labels $y_{i} \in$ $\{1,2, \ldots, C\}$, where $C$ is the number of classes, and $n$ is the total number of training samples. Let $n_{j}$ be the number of available training samples for the $j$ th class, such that $\sum_{j=1}^{C} n_{j}=n$. LDA seeks to find a linear transformation $W$ such that the within-class scatter is minimized and the between-class scatter is maximized. A common approach is to find a projection that maximizes Fisher's ratio of the determinant of $S^{(b)}$ to the determinant of $S^{(w)}$, i.e.,

$$
J_{1}(W)=\frac{\left|W^{\top} S^{(b)} W\right|}{\left|W^{\top} S^{(w)} W\right|} .
$$

In (1), $S^{(b)}$ is the between-class scatter matrix, and $S^{(w)}$ is the within-class scatter matrix. Note that the total scatter matrix $S^{(t)}$ is the sum of $S^{(b)}$ and $S^{(w)}$.

A popular solution to the SSS problem within the pattern recognition community is to employ PCA as an intermediate unsupervised dimensionality reduction projection prior to applying typical LDA transformation, i.e., the SLDA approach [1], [4]. In [10], KPCA (a kernel version of PCA) followed by LDA (called KSLDA) was introduced and studied for face- and handwritten-digit recognition. KPCA is first implemented via an eigendecomposition problem in a kernel space produced by nonlinear mapping $\Phi$. Traditional LDA is then performed in this KPCA-induced feature space.

Previously, NA-PCA (or MNF) has been proposed to provide a noise-robust dimensionality reduction for hyperspectral data. In [7], Nielsen et al. proposed kernel MNF (KMNF), which is a straightforward kernel extension of MNF. In KMNF, noise is directly estimated in the kernel space, and then a transformation maximizing the signal covariance while minimizing the noise covariance is estimated.

In this letter, we propose improved LDA algorithms that operate in either a NA-PCA subspace [6] or a noise-adjusted KPCA (NA-KPCA) subspace [7], i.e., NA-SLDA and NAKSLDA, respectively. The first step of NA-SLDA consists of an unsupervised projection (NA-PCA) that seeks to maximize SNR in the projected subspace, whereas the second step consists of a projection that maximizes class separation as measured by Fisher's ratio (i.e., LDA). By operating in a lower dimensional subspace, the NA-SLDA formulation is expected to be stable even when the input dimensionality $d$ of the feature space is high (e.g., as is the case for HSI), as well as when the number $n$ of training samples is small. For the proposed NAKSLDA algorithm, NA-KPCA first seeks to maximize SNR in a kernel-induced space, and a traditional LDA projection is performed on this NA-KPCA subspace. It is worthwhile to note that the difference between traditional KDA and KSLDA is that the former is accomplished by implementing traditional LDA directly in a kernel-induced feature space, whereas LDA is applied on the KPCA-induced feature space in the latter.

\section{NA-SLDA AND NA-KSLDA}

PCA projects input data onto a lower dimensional subspace, which maximizes the variance of the data in the projected subspace. Let $\Sigma$ be the data covariance matrix estimated from samples $\mathbf{X}=\left\{\mathbf{x}_{i}\right\}_{i=1}^{n}$ in $\mathbb{R}^{d}$. Assume that $V$ is the eigenvector matrix of $\Sigma$, such that $V^{\top} \Sigma V=\Lambda$, where $\Lambda$ is the eigenvalue matrix. Then, the PCA projection is

$$
\mathbf{Y}_{\mathrm{PCA}}=V^{\top}(\mathbf{X}-\mathbf{M})
$$

where $\mathbf{M}=\mathbf{m} \cdot \mathbf{1}, \mathbf{m}=\frac{1}{n} \sum_{i} \mathbf{x}_{i}$, and $\mathbf{1}=\left[\begin{array}{lll}1 & \cdots & 1\end{array}\right]$. Note that $\mathbf{m}$ is of size $d \times 1,1$ is a $1 \times n$ row vector of all ones, and $\mathbf{M}$ is of size $d \times n$.

Instead of variance, NA-PCA [5], [6] employs SNR as the optimization criterion. Specifically, let $\Sigma_{\text {noise }}$ be the covariance matrix of the noise and $F$ be a noise-whitening matrix such that $F^{\top} \Sigma_{\text {noise }} F=I$, where $I$ is the identity matrix. We then transform $\Sigma$ via $F$, i.e., $F^{\top} \Sigma F=\Sigma_{\text {noise_adj, }}$ such that $\Sigma_{\text {noise_adj }}$ is the covariance matrix after noise whitening. Find matrix $G$, such that $G^{\top} \Sigma_{\text {noise_adj }} G=I$. Then, the NA-PCA projections are calculated as

$$
\mathbf{Y}_{\mathrm{NA}-\mathrm{PCA}}=G^{\top} F^{\top}(\mathbf{X}-\mathbf{M}) .
$$

The challenge in effectively employing NA-PCA is accurately estimating the noise covariance matrix $\Sigma_{\text {noise. }}$ In previous work [6], the following method has been successfully employed for this task. Express $\Sigma$ as $\Sigma=D E D$, where $D=$ $\operatorname{diag}\left\{\left\{\sigma_{i}\right\}_{i=1}^{d}\right\}$, with $\sigma_{i}^{2}$ being the $i$ th diagonal elements of $\Sigma$ and $E$ being the correlation-coefficient matrix. Similarly, $\Sigma^{-1}$ can be also decomposed as $\Sigma^{-1}=D_{\Sigma^{-1}} E_{\Sigma^{-1}} D_{\Sigma^{-1}}$, where $D_{\Sigma^{-1}}=\operatorname{diag}\left\{\left\{\zeta_{i}\right\}_{i=1}^{d}\right\}$, with $\zeta_{i}^{2}$ being the $i$ th diagonal entry of $\Sigma^{-1}$. In [5], $\zeta_{i}$ was defined as $\zeta_{i}=\sigma_{i}^{-1}\left(1-r_{d-i}^{2}\right)^{-(1 / 2)}$, where $r_{d-i}^{2}$ is the multiple correlation coefficient of the $i$ th band on the other $d-i$ bands obtained via multiple regression theory. The advantage of using $\zeta_{i}$ instead of $\sigma_{i}$ is that the former removes its correlation with its peers, whereas the latter does not. Consequently, $\zeta_{i}^{2}$ is the reciprocal of the noise estimation for the $i$ th band, such that the noise covariance matrix $\Sigma_{\text {noise }}$ can be estimated as $\Sigma_{\text {noise }}=\operatorname{diag}\left\{\left\{\zeta_{i}^{-2}\right\}_{i=1}^{d}\right\}$.

We propose that employing the NA-PCA projection $\mathbf{Y}_{\mathrm{NA}-\mathrm{PCA}}$ as preprocessing to LDA would serve two important purposes: 1) The NA-PCA projection will discard the null space of $S^{(t)}$, making the LDA formulation tractable, even under SSS conditions; and 2) by employing NA-PCA instead of PCA, there will be added robustness to noise in the data. That is, NA-PCA is able to suppress noise from the input data by diagonalizing the noise covariance matrix $\Sigma_{\text {noise }}$. Based on the SNR criterion, the lower order (principal) components of the NA-PCA transformation are interpreted as representing useful signal information, whereas the higher order components are interpreted to represent noise. The final projection of the proposed NA-SLDA algorithm is

$$
\mathbf{Z}=W^{\top} \mathbf{Y}_{\mathrm{NA}-\mathrm{PCA}}
$$

where $W$ is the typical LDA transformation.

For classification tasks, such as ground-cover classification with HSI, NA-SLDA is expected to be significantly beneficial as a feature extraction strategy. The intermediate subspace alleviates any ill-conditioning due to SSS and high input-space dimensionality while providing robustness to noise, and the 
(a)

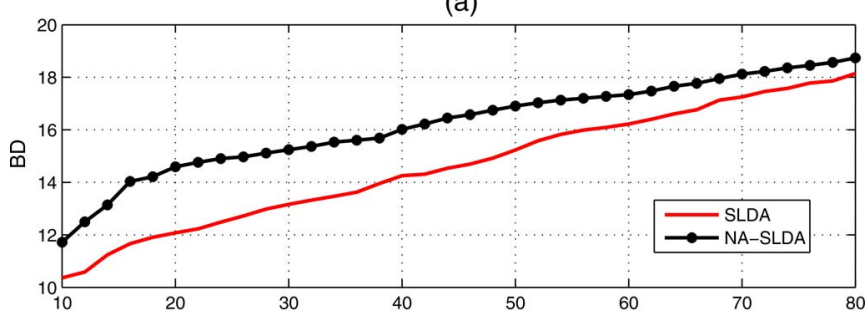

(b)

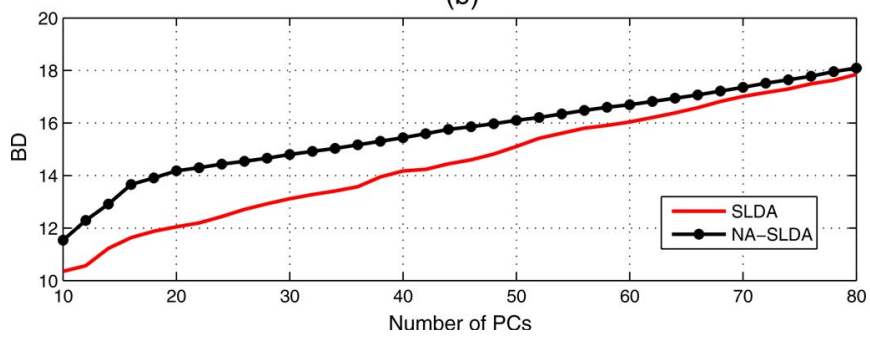

Fig. 1. Comparison of BD under different noise cases for the Indian Pines data set. (a) $\mathrm{SNR}=\infty$ and (b) $\mathrm{SNR}=35 \mathrm{~dB}$.

final LDA projection results in a lower dimensional feature space that retains the useful class-separation information. In order to show the effectiveness of NA-SLDA, we compare the Bhattacharyya distance (BD) [11] in the lower dimensional feature spaces of NA-SLDA and SLDA using the Indian Pines data introduced in Section IV. BD values measure the separability between classes, i.e., larger value indicates greater separability. Fig. 1 illustrates the average BD values among classes for the two dimensionality reduction techniques for two different values of the noise power. These results illustrate the noise robustness attained via the NA-SLDA transformation.

Next, the given algorithm is extended to its nonlinear kernelinduced counterpart. Consider a nonlinear mapping, i.e., $\Phi$ of the input data, such that $K_{\mathbf{x}_{i}, \mathbf{x}_{j}}=\left\langle\Phi\left(\mathbf{x}_{i}\right), \Phi\left(\mathbf{x}_{j}\right)\right\rangle$. Equations (2)-(4) are modified to become

$$
\begin{aligned}
\mathbf{Y}_{\mathrm{KPCA}} & =V_{\Phi}^{\top}\left(\mathbf{X}_{\Phi}-\mathbf{M}_{\Phi}\right) \\
\mathbf{Y}_{\mathrm{NA}-\mathrm{KPCA}} & =G_{\Phi}^{\top} F_{\Phi}^{\top}\left(\mathbf{X}_{\Phi}-\mathbf{M}_{\Phi}\right) \\
\mathbf{Z}_{\Phi} & =W^{\top} \mathbf{Y}_{\mathrm{NA}-\mathrm{KPCA}}
\end{aligned}
$$

respectively, where $\quad \mathbf{X}_{\Phi}=\left\{\Phi\left(\mathbf{x}_{i}\right)\right\}_{i=1}^{n}, \quad \mathbf{M}_{\Phi}=$ $\left[(1 / n) \sum_{i=1}^{n} \Phi\left(\mathbf{x}_{i}\right)\right] \cdot \mathbf{1}, \quad \mathbf{Z}_{\Phi}$ is the final projection, and $V_{\Phi}, G_{\Phi}$, and $F_{\Phi}$ are the corresponding matrices in the kernelinduced space for the proposed NA-KSLDA algorithm. In this letter, the radial-basis-function kernel [7] is used, such that $K_{\mathbf{x}_{i}, \mathbf{x}_{j}}=\exp \left(-\left(\left\|\mathbf{x}_{i}-\mathbf{x}_{j}\right\|^{2} / 2 \sigma^{2}\right)\right)$, where $\sigma$ is a user-defined parameter.

\section{EXPERIMENTS AND ANALYSIS}

We now empirically evaluate the classification performance of the proposed NA-SLDA and its kernel version, i.e., NAKSLDA, for hyperspectral data classification under noisy conditions. In this letter, we assume that the noise is independent of the signal information and in the form of white Gaussian noise of varying power added to the spectral bands, as was done in [12]. A quadratic Gaussian maximum-likelihood estimation classifier [2] is employed following all dimensionality reduction techniques in all results.

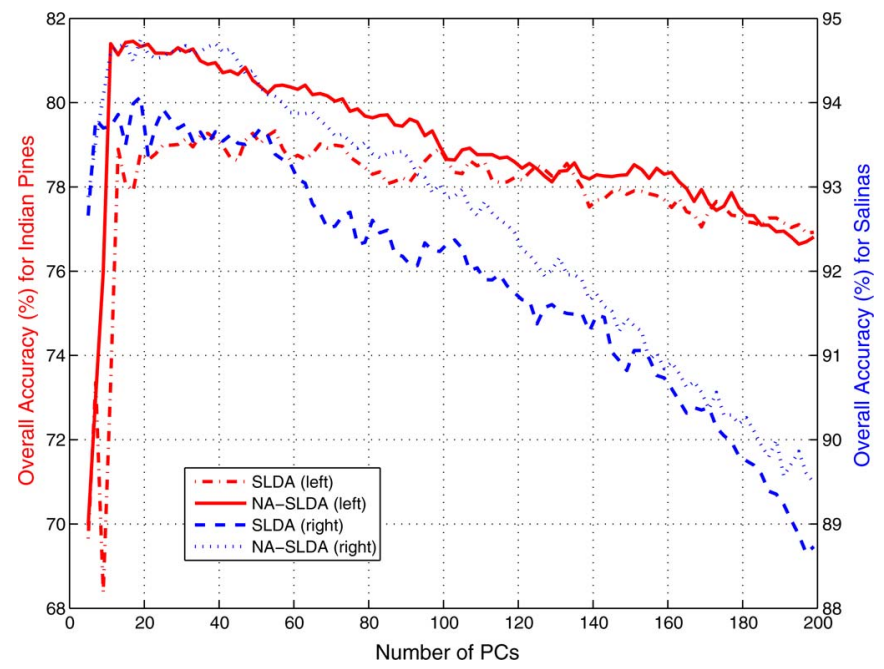

Fig. 2. Classification accuracy versus number of PCs of SLDA and NA-SLDA for two experimental data sets. Parameter tuning using limited training samples. Red: Indian Pines; blue: Salinas.

The first HSI data set employed was acquired using NASA's AVIRIS sensor ${ }^{1}$ and was collected over northwest Indiana's Indian Pine test site in June 1992. The image represents a vegetation classification scenario with $145 \times 145$ pixels and 220 spectral bands with a spatial resolution of $20 \mathrm{~m}$. There are 16 different land-cover classes in the original ground truth for this data set, whereas eight classes of interest are used in this letter. There are about 187 training samples per class and a total of 7100 testing samples (randomly chosen from the groundtruth map with no overlap between the training and testing sets).

We compare the classification performance of the proposed algorithms with popular approaches to address the issue of noise and the SSS issue for classification: 1) traditional kernel methods, i.e., KDA and SVM; 2) the popular combination of PCA and LDA in the form of SLDA; and 3) the combination of KPCA and LDA in the form of KSLDA. We also consider LDA used alone without any dimensionality reduction preprocessing. The dimensionality after the LDA transformation is $C-1$, where $C$ denotes the number of classes identified in the data set. The numerical values of the optimal parameter $\sigma$ for KDA, SVMs, and KSLDA after tuning using experimental training data are $0.1,0.2$, and 0.5 , respectively.

For parameter tuning, one can employ leave-one-out cross validation. In our work, we split the available training samples into further development data; more specifically, the available training samples are partitioned into two groups called "development data for training" (approximate 500 samples) and "development data for testing" (900 samples). We use these two groups of development data for parameter tuning to obtain the system with superior classification performance. Fig. 2 plots the overall development data accuracy versus the number of HSIs (PCs) for SLDA and NA-SLDA (red lines). The optimal number of PCs is around 40 for SLDA and 20 for NA-SLDA. For KSLDA and NA-KSLDA (KPCA and NA-KPCA are used as preprocessing), the size of the kernel matrix is determined by

\footnotetext{
${ }^{1}$ http://www.ehu.es/ccwintco/index.php/Hyperspectral_Remote_Sensing_ Scenes
} 


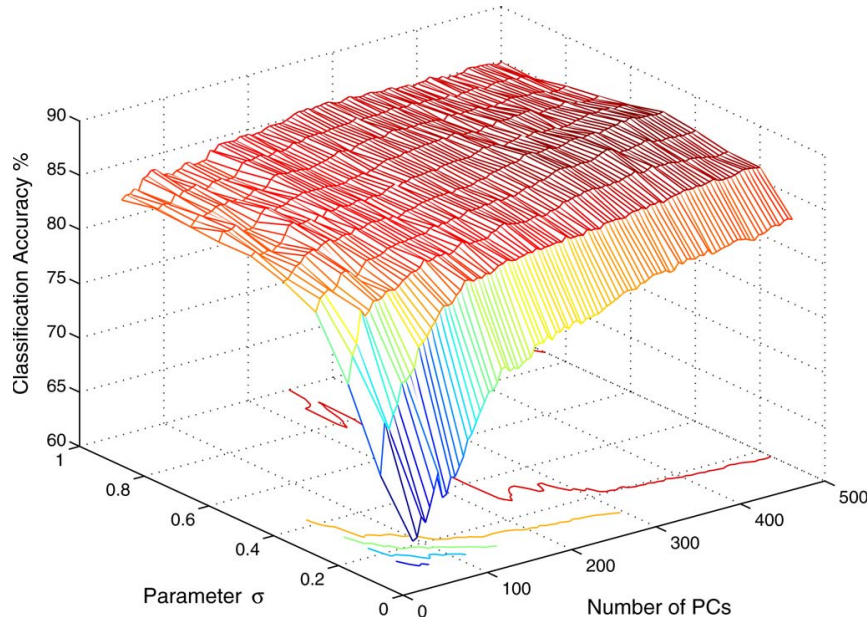

Fig. 3. Classification accuracy versus number of PCs as well as parameter $\sigma$ of NA-KSLDA for the Indian Pines data set. Parameter tuning using limited training samples.

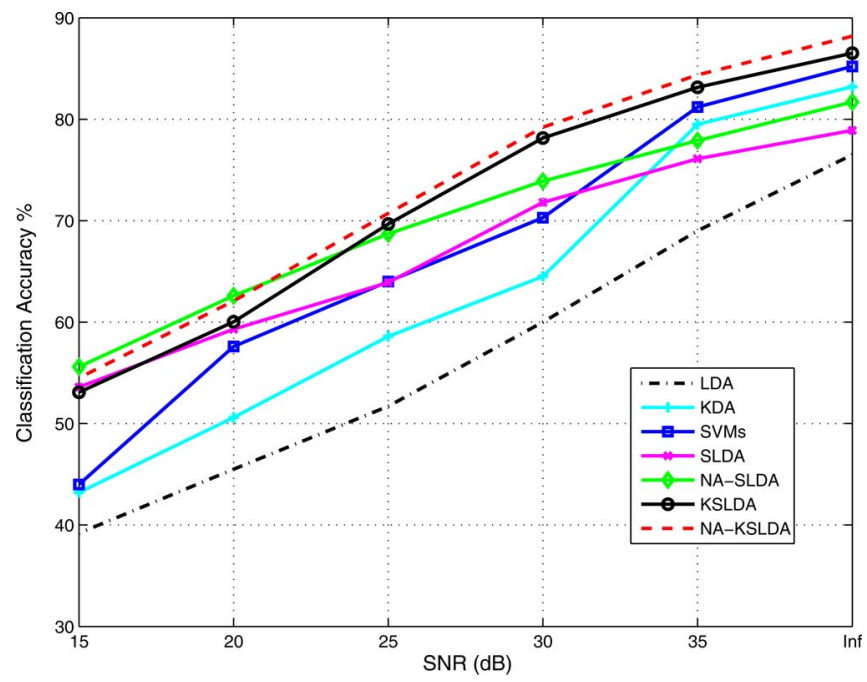

Fig. 4. Classification accuracy versus SNR for the Indian Pines data set.

the number of training samples. Fig. 3 illustrates the classification accuracy versus the number of PCs, as well as the kernel parameter $\sigma$. From this experiment, we infer the optimal $\sigma$ to be 0.5 and have found that, when the number of PCs increases to around 200, the classification accuracy tends to be stable. These figures are used to find the appropriate number of PCs (i.e., that which maximizes the classification accuracy for the technique and the data set at hand) and parameter $\sigma$. We note that, while we report results corresponding to just one SNR for the hyperspectral data, similar results were observed at other SNRs.

Fig. 4 shows the classification accuracy versus SNR for the Indian Pines data set. It can be seen in the figure that the performance of NA-KSLDA is superior to that of others, yielding higher classification accuracy over a range of SNRs. Baseline algorithms, such as SVMs, work well in low-noise situations. However, under severe noise, SVM performance is even worse than SLDA and NASLDA wherein PCA preprocessing can suppress some of the noise. Furthermore, in many practical situations, the number of available training samples is often insufficient for effectively training the classifier. Fig. 5 depicts the overall

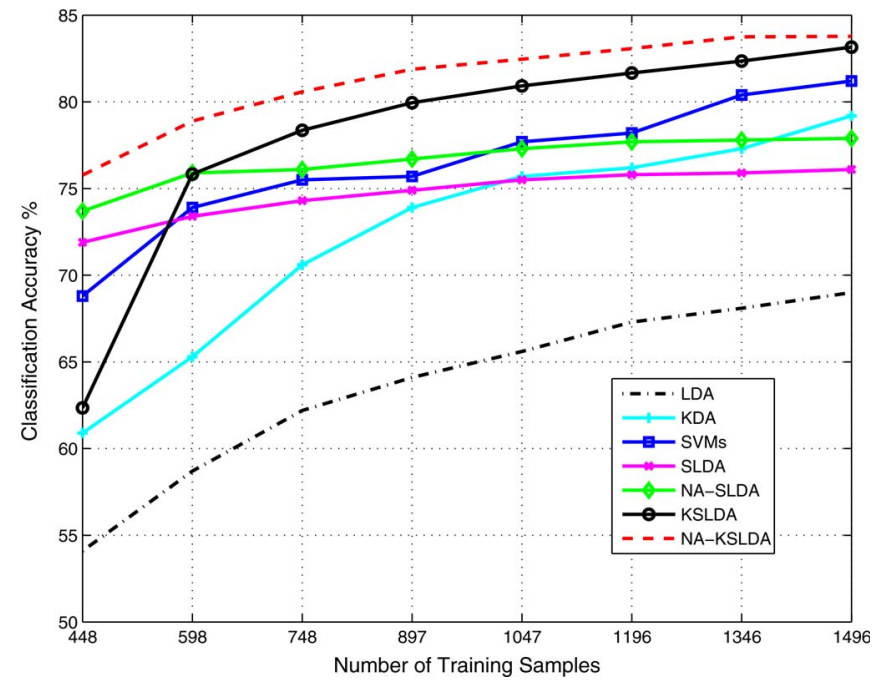

Fig. 5. Indian Pines data set. Effect of number of training samples for noise $\mathrm{SNR}=35 \mathrm{~dB}$.

classification accuracy as a function of the numbers of training samples employed. It can be seen that NA-KSLDA offers the most robustness to the SSS problem, i.e., NA-KSLDA provides superior classification performance even when very few training samples are employed. Hence, we conclude that it is an effective feature extraction strategy for HSI classification tasks.

Additionally, Fig. 6 depicts thematic maps resulting from the classification of the Indian Pines data set using NA-KSLDA, NA-SLDA, and SLDA. We produced ground-cover maps of the entire HSI scene for these techniques. Consistent with the previous observations, we see that NA-KSLDA provides the most reliable and the least noisy classification map.

The second data set employed was collected by an AVIRIS sensor over Salinas Valley, CA, USA. The area covered comprises $512 \times 217$ pixels with a spatial resolution of $3.7 \mathrm{~m}$ and includes vegetation and bare soil, as well as vineyard fields. The ground truth contains 16 classes. There are about 30 chosen training samples per class and 470 testing samples per class in our experiment. Fig. 2 illustrates the parameter tuning process for SLDA and NA-SLDA (blue lines) using these data. The optimal number of PCs is around 20 for the two techniques. Table I shows the classification results of different algorithms for two cases, i.e., without adding noise $(\mathrm{SNR}=\infty)$, and $\mathrm{SNR}=$ $35 \mathrm{~dB}$. For the noiseless case, the classification accuracy values of KDA and SVM are $91.45 \%$ and $94.39 \%$, respectively; for an SNR of $35 \mathrm{~dB}$, the results of KDA and SVM are $90.21 \%$ and $92.35 \%$, respectively. All algorithms were implemented under optimal parameters obtained via limited training samples. From the perspective of classification performance, we observe a similar performance trend, as observed with the first data set employed earlier.

We also compare the computational complexity of the classification methods. All the experiments are carried out using Matlab on an Intel(R) Core(TM)2 Duo CPU machine with 4 GB of RAM. Except for LDA, all the algorithms were implemented using SVM and Kernel Methods Toolbox. ${ }^{2}$ As an example, the execution times for the Salinas data set are shown in

\footnotetext{
${ }^{2} \mathrm{http} / / /$ asi.insa-rouen.fr/enseignants/ arakotom/toolbox/index.html
} 


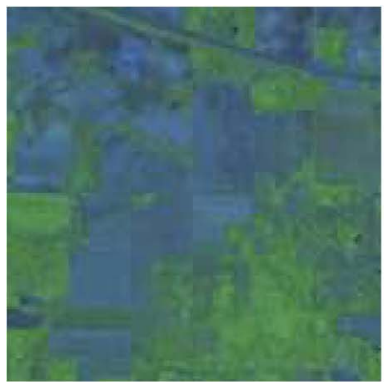

(a) False-color original

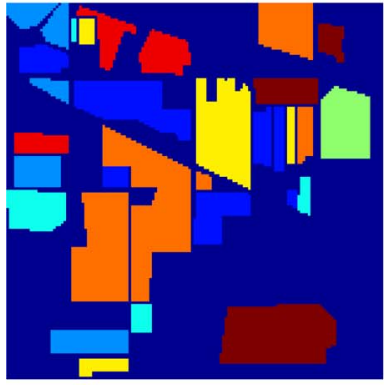

(b) Ground-truth map

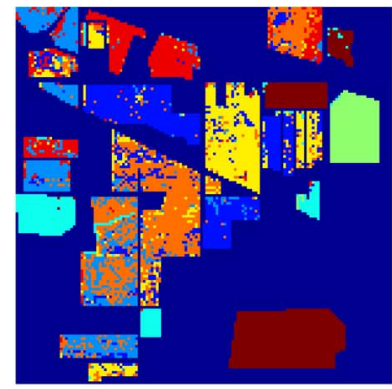

(c) SLDA, SNR $=35 \mathrm{~dB}$

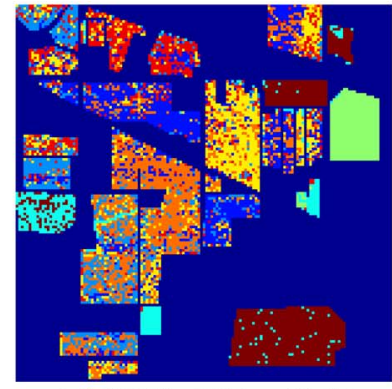

(d) SLDA, SNR $=20 \mathrm{~dB}$

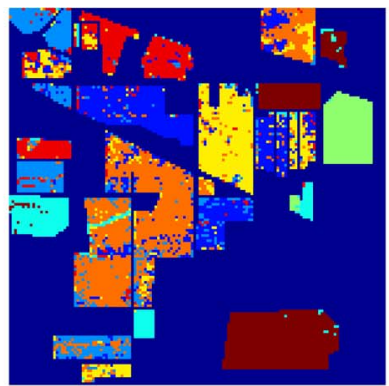

(e) NA-SLDA, SNR $=35 \mathrm{~dB}$

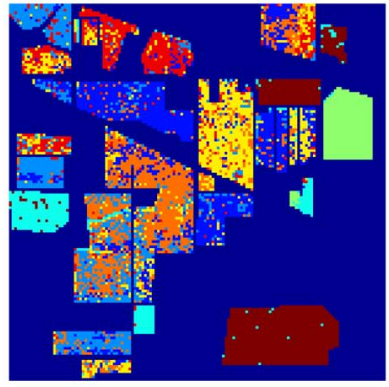

(f) NA-SLDA, SNR $=20 \mathrm{~dB}$

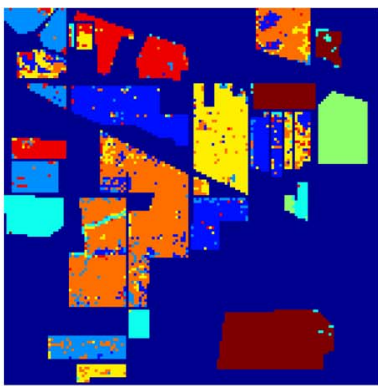

(g) NA-KSLDA, SNR $=35 \mathrm{~dB}$

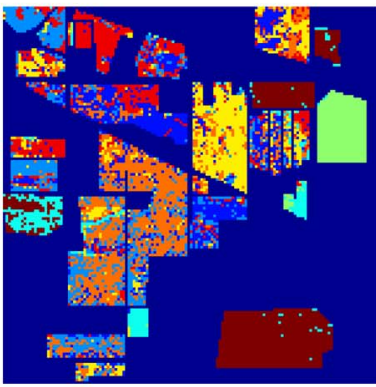

(h) NA-KSLDA, SNR $=20 \mathrm{~dB}$

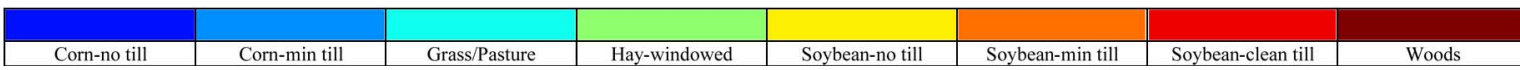

Fig. 6. Classification maps with two different SNRs for the Indian Pines data set. (a) False color image using bands 60, 40, and 20. (b) Ground truth of the labeled area with eight classes. (c)-(h) Thematic maps resulting from classification. (a) False-color original. (b) Ground-truth map. (c) SLDA, SNR $=35 \mathrm{~dB}$. (d) SLDA, SNR $=20 \mathrm{~dB}$. (e) NA-SLDA, SNR $=35 \mathrm{~dB}$. (f) NA-SLDA, SNR $=20 \mathrm{~dB}$. (g) NA-KSLDA, SNR $=35 \mathrm{~dB}$. (h) NA-KSLDA, SNR $=20 \mathrm{~dB}$.

TABLE I

Overall ACCURACy (\%) AND THE COMPUtational Cost For Different Algorithms Using THe SALinas Data SET

\begin{tabular}{|c|c|c|c|c|c|}
\cline { 2 - 6 } \multicolumn{1}{c|}{} & \multicolumn{5}{c|}{ Algorithms } \\
\hline Noise SNR & LDA & SLDA & NA-SLDA & KSLDA & NA-KSLDA \\
\hline$\infty$ & 87.12 & 94.04 & 95.44 & 94.57 & 96.34 \\
\hline $35 d B$ & 81.56 & 92.22 & 93.18 & 93.08 & 93.51 \\
\hline \hline Time $(s)$ & 32.5 & 29.5 & 29.9 & 34.0 & 35.7 \\
\hline
\end{tabular}

Table I. The run times of KDA and SVM are 32.4 and $4.37 \mathrm{~s}$, respectively.

\section{CONCLUSION}

In this letter, we proposed new feature extraction methods, i.e., NA-SLDA and its kernel version NA-KSLDA, for HSI classification. By employing NA-PCA or NA-KPCA as preprocessing to standard LDA, the NA-SLDA and NA-KSLDA approaches resulted in superior classification performance even under SSS and noisy conditions. This was as expected since both NA-PCA and NA-KPCA use SNR (instead of simply the variance as in PCA/KPCA) as the optimization criterion that drives the projection of the data onto a lower dimensional subspace. The AVIRIS Indian Pines hyperspectral data set was used to evaluate the proposed method and compare it to traditional feature extraction methods. Experimental results demonstrated that the proposed NA-KSLDA approach provides better classification performance as compared with traditional LDA and SLDA, as well as to KDA and SVMs, i.e., both under low SNR and SSS conditions. We note that the noise covariance for NA-PCA has been estimated using the approach developed specifically for AVIRIS HSI in [8]. In subsequent work, we plan to investigate the estimation of the noise covariance matrix for other data sets and further apply into our proposed classification strategy for these data.

\section{REFERENCES}

[1] A. M. Martinez and A. C. Kak, "PCA versus LDA," IEEE Trans. Pattern Anal. Mach. Intell., vol. 23, no. 2, pp. 228-233, Feb. 2001.

[2] S. Prasad and L. M. Bruce, "Limitations of principal component analysis for hyperspectral target recognition," IEEE Geosci. Remote Sens. Lett., vol. 5, no. 4, pp. 625-629, Oct. 2008.

[3] K. Das and Z. Nenadic, "An efficient discriminant-based solution for small sample size problem," Pattern Recognit., vol. 42, no. 5, pp. 857-866, May 2009.

[4] J. Yang and J. Yang, "Why can LDA be performed in PCA transformed space?" Pattern Recognit., vol. 36, no. 2, pp. 563-566, Feb. 2003.

[5] C. I. Chang and Q. Du, "Estimation of number of spectrally distinct signal sources in hyperspectral imagery," IEEE Trans. Geosci. Remote Sens., vol. 42, no. 3, pp. 608-619, Mar. 2004.

[6] Q. Du, W. Wei, D. May, and N. H. Younan, "Noise-adjusted principal component analysis for buired radioactive target detection and classification," IEEE Trans. Nucl. Sci., vol. 57, no. 6, pp. 3760-3766, Dec. 2010.

[7] A. A. Neilsen, "Kernel maximum autocorrelation factor and minimum noise fraction transformations," IEEE Trans. Image Process., vol. 20 no. 3, pp. 612-624, Mar. 2011.

[8] R. E. Roger and J. F. Arnold, "Reliably estimating the noise in AVIRIS hyperspectral images," Int. J. Remote Sens., vol. 17, no. 10, pp. 19511962, 1996.

[9] W. Li, S. Prasad, J. E. Fowler, and L. M. Bruce, "Locality-preserving discriminant analysis in kernel-induced feature spaces for hyperspectra image classification," IEEE Geosci. Remote Sens. Lett., vol. 8, no. 5 , pp. 894-898, Sep. 2011.

[10] J. Yang, A. F. Frangi, J. Yang, D. Zhang, and Z. Jin, "KPCA plus LDA: A complete kernel Fisher discriminant framework for feature extraction and recognition," IEEE Trans. Pattern Anal. Mach. Intell., vol. 27, no. 2 pp. 230-244, Feb. 2005.

[11] C. I. Chang and S. S. Chiang, "Anomaly detection and classification for hyperspectral imagery," IEEE Trans. Geosci. Remote Sens., vol. 40, no. 6 , pp. 1314-1325, Jun. 2002.

[12] G. Chen and S. E. Qian, "Denoising of hyperspectral imagery using principal component analysis and wavelet shrinkage," IEEE Trans. Geosci. Remote Sens., vol. 49, no. 3, pp. 973-980, Mar. 2011. 